\title{
Änderungen unvermeidlich
}

\section{Stellungnahme zur Novelle der Approbationsordnung.}

Der FVDZ hat bereits nach der Vorlage des ersten Entwurfs durch das BMG eine Stellungnahme vorgelegt, in der die wichtigsten Kritikpunkte an der A0Z-Novelle aufgelistet werden. Eine grundlegende Forderung des FVDZ bezieht sich auf die Finanzierung der zahnmedizinischen Ausbildung.

AUTOR: PD DR. THOMAS WOLF

$\triangle$ Eine Erhöhung des Ausbildungsaufwandes um zirka sechs Prozent ist vorgesehen. Diese soll mit einer Reduzierung der Studierendenzahl um etwa sechs Prozent (126 Studienplätze) einhergehen. Der prozentual wachsende Anteil weiblicher Studierender läßt ein verändertes Berufsbild der Zahnmedizin in Deutschland erwarten. Dem vielmals durch etliche Autoren beschriebenen Unterschied zu bisherigen Arbeitszeitmodellen und Teilzeit- beziehungsweise Angestelltentätigkeiten mit möglichen veränderten oder verkürzten Lebensarbeitszeiten muss Rechnung getragen werden. Eine Reduzierung der Studierendenzahl ist demnach nicht empfehlenswert.

$\triangle$ Die geplante Verbesserung der Betreuungsrelation ist sehr erfreulich. Allerdings ist diese nicht kostenneutral umsetzbar. Eine wie im Entwurf formulierte Reduzierung der Behandlungszeit führt für Patienten nicht zu einer attraktiveren Behandlung, da sich das Tempo im Behandeln der Studierenden nicht durch Halbieren der Behandlungszeit verdoppelt. Durch integrierte Kurse, die inhaltlich zu befürworten sind, die Betreuungsrelation $\mathrm{zu}$ verbessern, indem man das Personal von bisher zwei klinischen Kursen zusammengelegt, würde für die Studierenden in einer verkürzten Behandlungszeit resultieren, denn ohne zusätzliche Personalmittel wäre dies nicht kostenneutral realisierbar. Einbußen der Qualität der Ausbildung wären die Folge. Wir regen daher an, zusätzliche Mittel zur Verfügung zu stellen.

$\triangle$ Wir begrüßen ausdrücklich die Durchführung zusätzlicher schriftlicher Prüfungen im Zuge der 1. und 3. Zahnärztlichen Abschnittsprüfung. Diese lassen objektivierbare, reproduzierbare und standardisierte Prüfungen zu. Allerdings ist der zusätzliche Prüfungsaufwand ebenso nicht kostenneutral umsetzbar, und es müssen zusätzliche Mittel zur Verfügung gestellt werden. Bedenken hat der FVDZ auch in Fragen der Angleichung der ersten vier Studiensemester von Human- und Zahnmedizinern.

$\triangle$ Allerdings möchten wir zu bedenken geben, dass dies Tor und Tür öffnet für Studiengangwechsel von der Zahn- in die Humanmedizin, was ohnehin bereits stattfindet. In der Folge würde hierdurch der Wechsel zusätzlich erleichtert. Der aktuell noch höhere Numerus clausus (NC) der Zahnmedizin bietet daher bereits jetzt schon die Möglichkeit, die viele Studierende nutzen, um über einen Studienplatz der Zahnmedizin einen gewünschten Studienplatz der Humanmedizin durch Wechsel zu erlangen. Bei einer weiter steigenden Zahl an Studiengangwechslern könnte in der Folge eine nicht ausreichende Anzahl ausgebildeter Zahnärzte und somit ein Engpass in der Versorgung in Deutschland entstehen.

Der Erhalt des Abschlusses „Staatsexamen" ist unseres Erachtens sehr wichtig, um auch die hohe Qualität der Ausbildung an Universitäten namentlich als Qualitätsmerkmal aufrechtzuerhalten. Dem Studiengang Zahnmedizin ist bisher ein Leistungspunktsystem fremd. Anwendung findet ein sogenanntes Leistungspunktsystem in den Bachelor/Master-Studiengängen. Die Erfahrung hat gezeigt, dass in vielen Studiengängen der Umstieg auf Bachelor/Master nach den Bologna-Kriterien die Gefahr einer Nivellierung der qualitativen Ausbildung birgt und sich ein Markt erschließt, der von privaten Investoren für aus dem Boden sprießende Modellstudiengänge in Europa führt, der in Zukunft zu deutlicher Überversorgung führen kann. Wir bitten dies zu berücksichtigen und empfehlen im Hinblick auf eine qualitativ hochwertige zahnärztliche Ausbildung, den Abschluss Staatsexamen beizubehalten. 